\title{
SNS EXTRACTION FAST KICKER SYSTEM DEVELOPMENT *
}

\author{
W. Zhang ${ }^{5}$, J. Sandberg, R. Lambiase, Y.Y. Lee, R. Lockey, J. Mi, T. Nehring, C. Pai, N. Tsoupas, \\ J. Tuozzolo, D. Warburton, and J. Wei \\ Brookhaven National Laboratory, Upton, NY 11973 \\ K. Rust, and R. Cutler \\ Oak Ridge National Laboratory, Oak Ridge, TN 37831
}

\section{Abstract}

The SNS Extraction Fast Kicker System is a very high power, high repetition rate pulsed power system. It was design and developed at Brookhaven National Laboratory. This system will consist of fourteen identical high voltage, high current modulators, and their auxiliary control and charging systems. The modulators will drive fourteen extraction magnet sections located inside of the SNS accumulator ring. The required kicker field rise time is $200 \mathrm{~ns}$, a pulse flattop of $700 \mathrm{~ns}$, a pulse repetition rate of 60 pulse-per-second. A 2500 Ampere per modulator output is required to reach the extraction kicker magnetic field strength. This design features a Blumlein PulseForming-Network based topology, a low beam impedance termination, a fast current switching thyratron, and low inductance capacitor banks. It has a maximum charging voltage of $50 \mathrm{kV}$, an open circuit output of $100 \mathrm{kV}$, and a designed maximum pulsed current output of $4 \mathrm{kA}$ per modulator. The overall system output will be multiple GVA with 60 Pulse-per-second repetition rate. A prototype modulator has been successfully built and tested well above the SNS requirement. The modulator system production is in progress.

\section{INTRODUCTION}

The Spallation Neutron Source accumulator ring will operate at fixed energy of $1.0 \mathrm{GeV}, 60 \mathrm{~Hz}$ repetition rate, with an average 1.5 MW beam power in ring. It is designed to compress the $1 \mathrm{~ms}$ beam pulse containing $1.6 \times 10^{14}$ protons to $650 \mathrm{~ns}$ for delivery onto target. Its configuration has OMEGA geometry. The lattice structure is a four-fold symmetry with four FODO arcs and four long straight sections. Each of the four dispersion free straight sections is for injection, extraction, RF, or beam collimation [1].
The fast beam extraction will be a one-turn, two-step process. A set of fourteen full-aperture kickers will eject the beam vertically from the accumulator ring into the extraction septum gap. The septum will further deflect it by $16.8^{\circ}$ into Ring to Target Beam Transport Line. Additional straight section space is reserved for two more extraction kicker modules to allow $1.3 \mathrm{GeV}$ upgrade.

The fast extraction kicker magnet and the extraction septum magnet are to be located in the extraction straight section. Seven of the extraction kicker magnet sections will be at up stream of a vertical focusing, horizontal defocusing narrow quadrupole doublet, and seven down stream of it. The extraction septum will be located $7 \mathrm{ft}$ down stream of the last kicker magnet section.

The SNS accumulator ring has a circumference of 248.0 meter. At $1.0 \mathrm{GeV}$, the beam resolution time is $945.4 \mathrm{~ns}$. The rise time of the kicker field must be less than the reserved beam gap of $250 \mathrm{~ns}$, to extract beam cleanly. The beam acceptance of the extraction region is $480 \pi-\mathrm{mm}$ during beam accumulation, and $400 \pi$-mm during beam extraction. Therefore a very large kicker magnet aperture is required. The main parameter specifications are listed in Table 1.

Table 1. Updated main parameter specification.

\begin{tabular}{|l|l|}
\hline Beam Rigidity & $5.6575 \mathrm{~T}-\mathrm{M}$ \\
\hline Extraction Energy & $1.0 \mathrm{GeV}$ \\
\hline Extraction type & Single-turn \\
\hline Magnet window & Full aperture \\
\hline Beam revolution period & $945.4 \mathrm{~ns}($ at $1.0 \mathrm{GeV})$ \\
& $911.1 \mathrm{~ns}$ (at $1.3 \mathrm{GeV})$ \\
\hline Beam gap during extraction & $250 \mathrm{~ns}$ \\
\hline Bunch length (full) & $645 \mathrm{~ns}$ \\
\hline Maximum extraction rate & $60 \mathrm{~Hz}$ \\
\hline Pulse flat-top length & $>700 \mathrm{~ns}$ \\
\hline Pulse Flat-top tolerance & $+1-3 \%$ \\
\hline Pulse rise time & $200 \mathrm{~ns}(1 \%-95 \%)$ \\
\hline Pulse fall time & $<16.6 \mathrm{~ms}$ \\
\hline
\end{tabular}

\footnotetext{
* SNS is managed by UT-Battelle, LLC, under contract DE-AC05-00OR22725 for the U.S. Department of Energy. SNS is a partnership of six national laboratories: Argonne, Brookhaven, Jefferson, Lawrence Berkeley, Los Alamos, and Oak Ridge.

ร email: arling@bnl.gov
} 


\begin{tabular}{|l|l|}
\hline Kicker strength & $\begin{array}{l}1.276 \text { to } 1.775 \mathrm{mrad} \\
\text { per section }\end{array}$ \\
\hline Total deflection strength & $20.344 \mathrm{mrad}$ \\
\hline Kicker horizontal aperture & $120 \mathrm{~mm}$ to $211.3 \mathrm{~mm}$ \\
\hline Kicker vertical aperture & $166 \mathrm{~mm}$ to $243 \mathrm{~mm}$ \\
\hline Kicker length & $\begin{array}{l}390 \mathrm{~mm} \text { to } 505 \mathrm{~mm} \text { per } \\
\text { section }\end{array}$ \\
\hline Kicker magnet inductance & $\begin{array}{l}695 \mathrm{nH} \text { to } 789 \mathrm{nH} \text { per } \\
\text { section }\end{array}$ \\
\hline Operating voltage & $<35 \mathrm{kV}$ per section \\
\hline Operating current & $<2.5 \mathrm{kA}$ per section \\
\hline $\begin{array}{l}\text { Beam Impedance } \\
\text { Termination }\end{array}$ & $\sim 250 \mathrm{hm}$ \\
\hline
\end{tabular}

\section{CHALLENGING ISSUES}

The extraction fast kicker system is a very critical system of the SNS Accumulator Ring. Its primary design goal is to extract the high intensity proton beam cleanly from the accumulator ring in single-turn. To meet the design specification, many technical challenges have to be addressed.

The fast kicker systems are usually rated at or below a few hundred mega-watts peak power, due to the technical difficulties combined with the pulse speed, voltage, current, pulse repetition rate, pulse lifetime, precision pulse shape, and magnetic load requirement. The present design of SNS Extraction Fast Kicker System is one of the largest high power fast kickers in its category. Its combined output pulse power reaches several Giga-Watts peak, an order of magnitude higher than usual.

Among many physical and technical challenges, the uncontrolled beam loss is the primary concern of the high intensity proton machine. The resistive impedance caused instability is one of the main factors attributed to beam loss. In the SNS ring design, various studies pointed out that the extraction kicker magnet inside the beam vacuum chamber is the leading impedance source [2] [3]. Therefore, to minimize the beam impedance of the extraction kicker is essential to the success of the SNS accumulator ring design.

The lumped magnet structure was chosen in the early stage of the SNS accumulator ring design for its structure simplicity and very high reliability. However, the beam impedance of the kicker magnets with open circuit structure, during beam accumulation, would dominate the overall beam impedance of the ring and the impedance caused beam loss.

As confirmed by the kicker magnet impedance measurement [4], the magnet impedance with open circuit has a very high resonance at $35 \mathrm{MHz}$ and is unacceptable. Even a $200 \mathrm{ohm}$ beam impedance termination is determined to be too high. A $25 \mathrm{ohm}$ to $50 \mathrm{ohm}$ beam impedance termination well matched to the modulator output pulse cable impedance is suggested to be the acceptable range. Note that, the short circuit beam impedance termination at the far end of the cable from the magnet is indeed not a good choice. This is not only because it is unfeasible, but also for its total mismatch to the cable impedance would cause $100 \%$ reflection and present rather high beam impedance.

To provide a low resistive beam impedance termination is at very high cost to the pulser design. The $25 \mathrm{ohm}$ resister at the output of the high voltage modulator, in parallel to the pulse transmission cable, will share half of the initial pulsed output current.

In addition, the beam extraction area is usually high in radiation level. To achieve high system maintainability and operability, the high voltage modulators have to be located outside the accumulator ring tunnel. This in general requires a factor of two voltage increase of the high voltage modulator output.

Another important specification of the SNS extraction fast kicker system is to be able to extract beam cleanly in the event of single module failure. This means a system redundancy and a large clearance at the septum gap. It further increases the deflection strength requirement of the fast kicker.

\section{SYSTEM OVERVIEW}

While utilizing the same industrial support as others, the new design has to meet all physical and technical specifications, within reasonable cost and schedule, and be feasible for engineering implementation. The task to provide such a design in an extremely short duration is very challenging.

\section{A. Main Schematic}

A design based on Blumlein pulser topology [7], as shown in Figure 1, was chosen for it can simultaneously satisfy all physical and technical requirements. The advantages of this design will be described in the later sections.

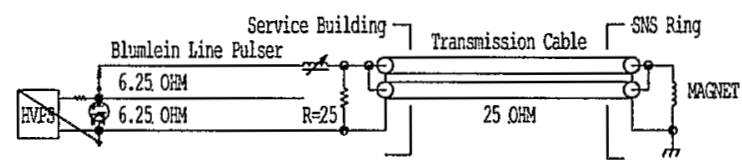

Figure 1. Simplified SNS extraction fast kicker schematic diagram.

\section{B. System Layout}

The SNS extraction fast kicker system will consist of fourteen high voltage modulators, fourteen kicker magnet sections, twenty-eight high voltage pulse transmission cables, and fourteen auxiliary and control systems, and a central trigger synchronization and distribution system. Each high voltage modulator will have an independent auxiliary and control system. It will be fully capable of independent remote and local operation. The high voltage modulators will be located in a service building outside of the accumulator tunnel. The output current pulses of each high voltage modulator will be transported to the corresponding kicker magnet section through two high voltage pulse cables. No active or dissipative components 
are used in the accumulator ring tunnel. The system layout is shown in Figure 2.

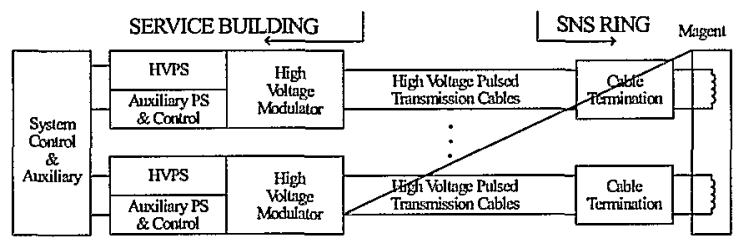

Figure 2. SNS Extraction Fast Kicker System Layout.

\section{Design Features}

In this design a Blumlein voltage doubler and a full reflection at kicker magnet to double the current provide a factor of four performance enhancement of the system. This design initially offered a very large design margin. Later, it was utilized by the physicists to enlarge the kicker magnet aperture to accommodate all four working points, further reduced beam impedance [5] [6], and increased beam acceptance at kicker magnets. Compare with the year 2001 parameters [7], the required total output power of the kicker modulators have to be increased by $86 \%$, and the margin of designed voltage over operating voltage shrink to $42 \%$ per modulator from $88 \%-173 \%$ per modulator.

During beam circulation, a saturable inductor made of a stack of ferrite rings will isolate Blumlein PFN structure from the $25 \mathrm{ohm}$ beam impedance termination, to ensure impedance matching to the transmission cable. This stack of ferrite rings will become saturated during high current conduction and can sharpen the output current pulse by suppressing the dark current.

With no active and dissipative components in the ring, the system is highly operable and maintainable. Also, in this design, only twenty-eight transmission cables are needed, largely reduced from conventional matched PFN design of more than ninety cables.

Another advantage of using the Blumlein based topology is that with lower charging and operating voltage, all major components are in the commercially available range. Although the switching current is doubled, it is well within the Hollow-anode thyratron switching capability. The typical output of the conventional PFN discharge induces a high to low transient, which stresses the output circuit. The initial output voltage transient of Blumlein pulser at the discharge will rise from zero to the charging level. For the same output pulse, the Blumlein pulser reduces the high voltage transient of the components. Thus, we were able to avoid very costly and time consuming development of high voltage pulsed power components such as pulse cables, connectors, feed-through, etc. Hence, the design is highly cost effective.

\section{HIGH VOLTAGE MODULATOR DEVELOPMENT AND TEST}

The Blumlein pulser can be implemented by two sets of high voltage cable pulse-forming lines or double deck of lumped L-C pulse forming networks. The later option was chosen for the compactness. We conducted design analysis, parameter optimization, and computer simulations to verify the design principle. Then, a proofof-principle model circuit was build and tested. Its output current waveform nicely matched computer simulation. Further, a full-scale prototype modulator has been designed, constructed, and tested at Brookhaven National Laboratory. Its test results successfully demonstrate the design concept.

Mechanical design of the high voltage pulse modulator provides many features for easy maintenance, such as, the modulator internal structure can be lifted out from its fluid container for device change and pulse shaping. Figure 3 shows the front view of the prototype modulator.

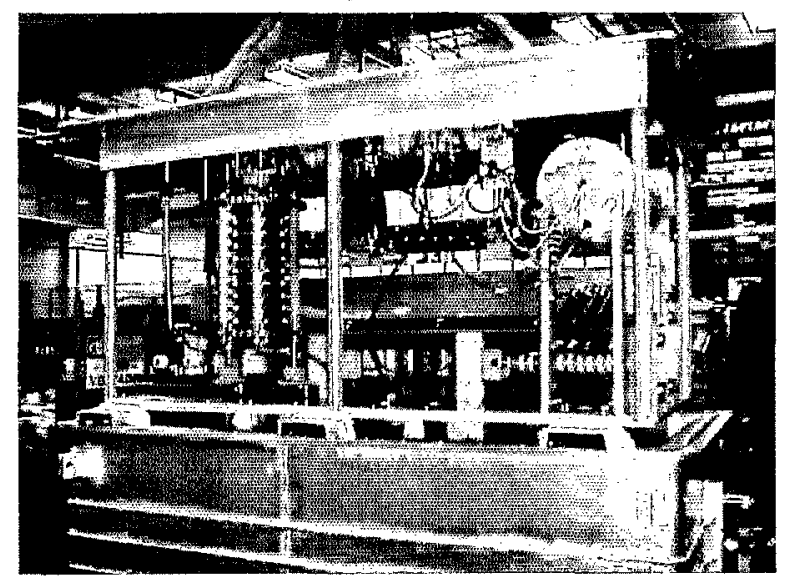

Figure 3. The SNS extraction fast kicker high voltage modulator prototype.

The Figures 4 and 5 show the side views of the prototype modulator. The E2V CX1925X thyratron is enclosed in a low inductance coaxial housing for fast current switching. The Blumlein Pulse Forming Network uses ultra low inductance high energy pulse capacitors developed by General Atomics.

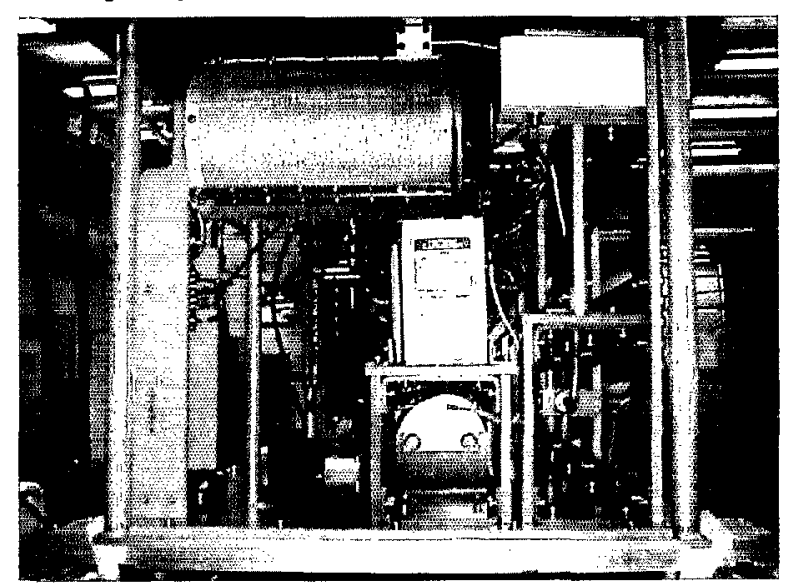

Figure 4. Modulator thyratron end side view. 


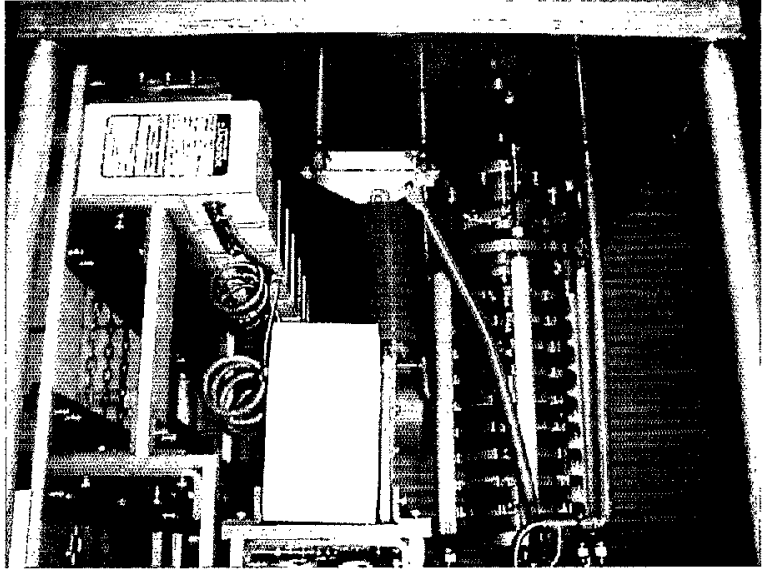

Figure 5. Modulator resistor end side view.

Varies tests have been conducted on the prototype modulator with the largest section of the kicker magnet load. The pulse transmission cables used in the test has a length of $600 \mathrm{ft}$. Accumulated high voltage test has reached 600 hours. For pulse shape verification and construction check, the unit was tested up to $40 \mathrm{kV}$ in air, in single shot mode. At $35 \mathrm{kV}$ full rated operation voltage, the prototype unit has been tested in silicon fluid at $60 \mathrm{~Hz}$ repetition rate for over 300 hours. A $40 \mathrm{KV}, 60 \mathrm{~Hz}$ repetition rate, 12 hours continuous accelerated lifetime test has been completed. And a $45 \mathrm{kV}, 30 \mathrm{~Hz}$ repetition rate, 8 hours continuous accelerated lifetime test has also been completed. A $50 \mathrm{kV}$ single shot mode test has been conducted. Figures 6 and 7 shows the kicker magnet current and current rise time waveforms at $50 \mathrm{kV}$.

Although the maximum charging voltage is $50 \mathrm{kV}$, we set the operation voltage limit of the modulator at below $35 \mathrm{kV}$ for the concerns of device lifetime, system reliability, fault tolerance, and system sustainability.

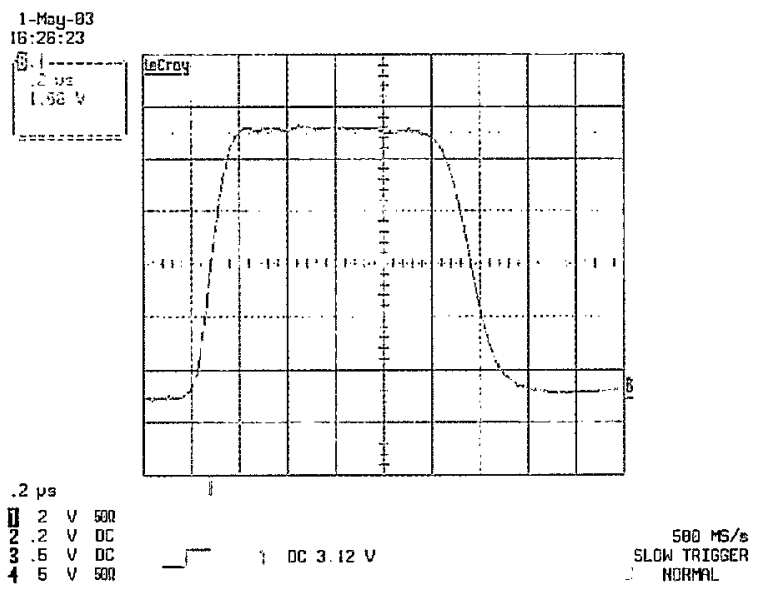

Figure 6. The magnet current waveform at $50 \mathrm{kV}$.

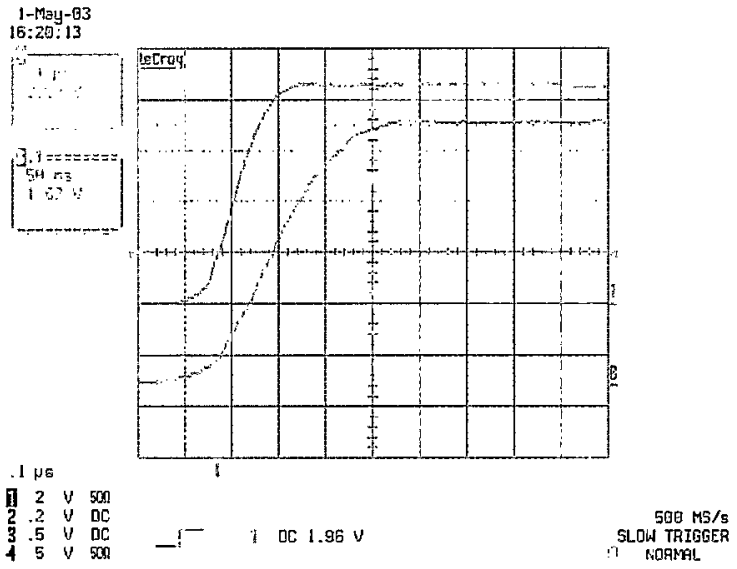

Figure 7. The kicker magnet current rise time waveform.

\section{CONCLUSION}

The prototype high voltage modulator has been designed, developed, and tested successfully. Its production contract has been awarded to Applied Power Systems last year, and the production has begun. We would like to express our appreciations to Mr. W.A. Morris of ISIS, Dr. R. Sheldrake of E2V, Mr. R. Cooper and Mr. R. Hartsock of General Atomics for their expert advice and technical support.

\section{REFERENCE}

[1]. J. Wei, et al., "Evolution of the Spallation Neutron Source Ring Lattice", 20TH ICFA Advanced Beam Dynamics Workshop, Chicago, IL, April 2002.

[2]. S.Y. Zhang, "SNS Storage Ring Impedances", BNL/SNS Technical Note No. 061, 1999.

[3]. S.Y. Zhang, "NSNS Transverse Instability", BNL/NSNS Technical Note No. 033, 1997.

[4]. D. Davino and H. Hahn, "Measurement and Analysis of the transverse coupling impedance of the SNS extraction kickers", BNL/SNS Technical Note No. $102,2001$.

[5]. D. Davino, et al., "Reduced Transverse Impedance of SNS Ring Extraction Kickers”, BNL/SNS Technical Note No. 112, Rev. A, 2002.

[6]. J. Wei, "Design and Optimization of the Spallation Neutron Source", Snowmass 2001.

[7]. W. Zhang, et al., "A New Conceptual Design of the SNS Full Turn Fast Extraction Kicker Power Supply System", Proceedings, 2001 Particle Accelerator Conference, June 2001, pp. 3714-3716.

[8]. W. Zhang, et al., "High Power Fast Kicker system for SNS Beam Extraction", Conference Record of the Twenty-Fifth International Power Modulator Symposium and 2002 High Voltage Workshop, June 30-July 3, 2002, pp 262-265. 